\title{
THE ASSESSMENT \\ OF EMOTIONAL COMPETENCE \\ IN NEURODEVELOPMENTAL DISORDERS THROUGH TEC I
} \author{
Nocerino Ludovica Cira ${ }^{5}$, Piperno Catiuscia ${ }^{6}$ \\ ${ }^{1}$ TNPEE, Centro Manzoni srl, Naples \\ ${ }^{2}$ TNPEE, University of Campania "L. Vanvitelli" \\ ${ }^{3}$ TNPEE, Centro Panda, Arzano \\ ${ }^{4}$ Neuropsichiatra Infantile, University of Campania "L. Vanvitelli" \\ ${ }^{5}$ Psicologa \\ ${ }^{6}$ Logopedista, Centro Medico Polispecialistico "Pianeta Salute” Bari
}

Martino Federica ${ }^{1}$, Cerroni Francesco ${ }^{2}$, Della Corte Giuseppina ${ }^{3}$, Carotenuto Marco ${ }^{4}$,

KEYWORDS: Emotions, emotional intelligence, emotional components and skills, neurodevelopmental disorders, TEC I (Test of emotion comprehension),

\section{ABSTRACT}

In the field of neurodevelopmental disorders, the use of a standardized assessment test - TEC I - has proven the complex emotional variability of each individual validating and expanding the theoretical psycho-affective assumptions (drawn from direct observation of subjects with typical development) in children belonging to different nosographic categories. The data obtained through singular and comparative analysis of the subjects not only promote new insights into the influence of the emotional sphere in the construction of personality and learning of typical and atypical growing children, but suggest the emergence of new rehabilitation procedures and therapeutic objectives to be achieved and undertaken

\section{INTRODUCTION}

The psycho-emotional dialectic is strictly dependent on the dynamic balance between the processes of self-regulation and the mechanisms of internalization and externalization of the logical-emotional flow of one's own and others' mental and affective state. In developmental age, the skills of reading, recognition and management of the emotional stimulus reflect the psychic and mental organization of the growing child and explain his future choices, behavioral and relational. The possibility of experiencing different emotions is not necessarily equivalent to knowing how to recognize, name or differentiate them, and expressing them does not mean consequently knowing how to manage, share or transmit them. The emotional stimulus can be recognized and not expressed (for example, contingent emotional-affective exchanges, prior to the development of language or present even in the absence of the same), expressed and not modulated (think of disruptive and maladaptive behaviors present in the presence of a correct, broad and varied psychological and emotional vocabulary or the use of an emotional labeling without communication or empathic exchange). To be emotionally competent means to have simultaneously integrated competences of self-regulation and analysis, to be able to look inside oneself and restructure oneself as one is not outside, to have an internal look and adapt it to the role undertaken, assumed or expected. This makes it possible to assert that there is no stopping phase in the development of emotional competencies, rather a continuous monitoring and constant adaptive flexibility of the emotional feedback invested or rediscovered. Continuous exposure to behavioral or cultural patterns, contextual demands, and the search for new stimuli, goals, and objectives to pursue allow for constant, dynamic, and mutually influential growth of attainable emotional and cognitive competencies.
Therefore, the analysis of the emotional components of a subject can be variable and diversified in temporal ranges changing in extension and frequency, regressing or evolving for different ways of thinking, living and acting.

\section{THEORETICAL ASPECTS Emotional development}

Since the neonatal period, the quality of psycho-affective experience - lived and experienced according to canons of emotional availability and/or relational disorganization - promotes the birth of a complex interpretative and behavioural system, equipped with precise processes of reception, elaboration, interpretation and analysis of data, habits and attitudes. According to Stern's psychodynamic thought, the dyadic intersubjective and contingent interactions of the first two years of life allow an active and dynamic knowledge of the internal and circumstantial world. In particular, the relational Self constructs the emotional and affective meaning of the world, systematically organizes lived experiences through continuous and variable attributions of mental and affective meanings collected in episodic, circumscribed and re-evocable memories. Interactive and emotional sequences derived from feedback and analysis processes are recorded, synthesized and mentalized progressively in:

- Mnestic traces or generalized representations (RIG);

- Mental Schemes or Operating Models (MOI).

The increasing developmental skills and competences, together with the-emotional meaning of the interactions promoted and undertaken as well as the environmental well-being in which they arise and are inserted, modify and update the mental schemes and mnestic representations. RIG and MOI will affect the future relational dynamics of the subject's life and will 
have a significant impact on the ability to connect with the internal world, tune in to the external one and understand thoughts, intentions, actions and emotions (ToM).

The synergy and stability of these internal representations forge precise psychic, behavioural and emotional profiles that can be categorized according to subjective and objective parameters, such as:

- Emotional quality of perceived and experienced environmental reality: Emotional tuning and feedback correlations affect the acquisition of relational skills.

- Social, cognitive, and language developmental skills:

- The child learns to perceive, identify and recognise self and other on both an individual and linguistic level, through the understanding of intentions, emotions, thoughts and mental and affective states of mind. The acquisition of speech supports exchanges, through the verbalization of requests or the declaration of implicit or latent needs.

- Hierarchically complex stages and processes of cognition: The analysis and general processing of primary and secondary environmental stimuli result in an interfering overview dependent on the stability of physiological and regulatory systems.

- Psycho-affective parental well-being. The psychological characteristics of the attachment figure are expressed and conveyed through the quality of care, attention and care offered. The disorganization of attachment, a symptom of psycho-affective instability, determines a significant collapse of the child's behavioral and attentional strategies (breakdown of psychic defenses). (breakdown of psychic defences).

- Parental educational styles and socio-cultural and value level.

- Familial and extra-familial organizations and care, marital relationships, routines, rituals and meanings in the home, belief systems and parental developmental histories (critical events and/or attachment dynamics, personality and temperament) associated with levels of permissiveness or solicitude, responsiveness or hostility condition the experiential possibility and quality of stimulation solicited or induced. The parental function of authoritative rather than authoritative, permissive rather than neglectful type is configured over time now as a protective factor now as a risk factor determining the socio-emotional development of the growing child (Belsky 1981).

The mentalization of emotions highlights a complex relationship between emotions and thoughts:-such that the emotional faculty can guide decisions, inhibit choices, allow logical thought or make it impossible. Emotions can evade control and take over powerfully, conversely the rational brain can assume a pre-dominant role in choosing which emotions to experience, hold back, share or disconnect (Golemann 1966).

To be emotionally valid subjects does not only mean to have developed one's emotional intelligence or to experience refuted emotions, but to practice the aptitude to reason with them.

Re-elaborating the different thoughts of numerous psychologists, the recognition and regulation of affective states and emotion-elicting social transitions can be said to be possible through:

- Introspective emotional skills (Skills perspective-taking);

- Sense of Self (one's self or ego identity);

- Moral sense (one's moral sense);

- Personal developmental history (one's development history).

Specifically, Emotional Skills cover:

- Awareness of one's own and others' emotional state (awareness of one's own emotions);

- Ability to discern and understand others' emotions;

- Use of appropriate emotional vocabulary or vocabulary (ability to use the vocabulary of emotions and expression);

- Empathic and sympathetic involvement (capacity for empathicinvolvement);

- Discrimination between internal emotional states and manifest emotional expressions (differentiating internal subjective emotional experience from external emotional expression);

- Use of behaviors facing diametrically opposed emotional situations and / or multiple valence ( $\mathrm{Ca}$ pacity for adaptivecoping with negative emotions);

- Qualitatively valid emotional communication (awareness of emotional communication within relationship);

- Emotional self-efficacy and/or self-control (capacity for emotional self-efficacy).

\section{Understanding emotions}

Pons and Harris, have analyzed the complex process of understanding emotions (emotion under standing) distinguishing 9 age-specific emotional components underlying. The components in their entirety determine the birth of an affective mind capable of attributing a wide range of meanings to internal/external events, as well as to mental, emotional and non-epistemic states divorced from beliefs, opinions and hypotheses. The hierarchical and complementary integration of these promotes over time the construction of a plastic, variable and continuously expanding self-regulatory repertoire. In particular:

1. Recognition and labeling;

2. Understanding external causes;

3. Understanding role of desires;

4. Understanding the role of beliefs;

5. Understanding the influence of memories;

6. Regulation of emotional experience;

7. Apparent/proven emotion distinction;

8. Categorization of mixed emotions;

9. Understanding the role of morality.

Both on a lexical and strategic-behavioural level, the child learns not only to recognise, identify and read in himself and in the other the manifest and hidden emotional state, but by analysing the emotional stimulus, he understands or predicts the behaviour related to it. Moreover, the authors insert and involve components in three dimensions concerning nature, cause and emotional control: the balance between components and interfered dimension sanctions different degrees of emotional awareness, knowledge and mastery. Specifically:

- Understanding the nature of emotions allows the analysis and recognition of emotional simultaneity and co-presentation, the first expressed through emotions of similar value directed towards a sin- 
General population tested with TEC-I

\begin{tabular}{l|lll}
\hline GROUP & DIAGNOSIS & CHAMPION & \% PERCENTAGE \\
Group $\boldsymbol{A}$ & Regulatory and Affective Immaturities & 23 & $46 \%$ \\
Group B & Genetic Syndromes & 6 & $12 \%$ \\
Group C & Language and DSA & 9 & $18 \%$ \\
Group D & MRI and SPMR & 6 & $12 \%$ \\
Group $\boldsymbol{E}$ & Dist. Social Adaptation & 6 & $12 \%$ \\
Total & & 50 & $100 \%$
\end{tabular}

Tab.1

gle object or event, the second through opposite emotions directed towards the same object or more objects and events. It arises through interpersonal experience with adults and peers and makes use of:

- mimic-expressive indexes (especially in the neonatal period and in early childhood);

○ psychological lexicon including emotional labels referring to mental states and experiences;

- Mentalization of complex states of mind (such as shame or guilt);

- emotional deductive hypothesis in conditions of conflict or discomfort.

- The understanding of the causes of emotions aris$e s$ at preschool age and allows the emergence of the causal link between events and emotions. We distinguish:

- external causes: corresponding to the relationship between observed actions and the emotional stimulus internal causes: globally expressed by the role of desires (access to the inner world of the subject), beliefs (on which the intensity and frequency of emotional reactions depend), memories and moral and ethical values.

- The understanding of emotion regulation arises through social exchange and availability and allows discrimination between expressed and actually felt emotion and understanding of the possibilities of ongoing emotional self-control (cuncurrent emotion).

The child understands that what is seen may not correspond to what is (misleading appearances or rules of exhibition) and that false belief may be related to the desire to obtain one's own advantages. The conscious use of coping strategies varies with age: initially the regulatory tools are behavioral, later psychological.

\section{METHODOLOGY AND MATERIALS \\ Sample Characteristics}

The administration of the TEC-I "Test of emotion comprehension" - Italian version of the Test of emotion comprehension - was addressed, through structured and standardized material and compliance with each protocol included in the manual, to a population of 50 children of sex and age between 4 and 11 years, in rehabilitation treatment of Neuro and Psychomo- tricity Therapy of the developmental age bi / three times a week in relation to medical prescription. For the purposes of the experimental study, the data collected were evaluated, calibrated and expressed anonymously and coded according to alphanumeric mode - as established by the privacy consent form signed in agreement with the parent of each subject minor. The population of individuals examined, has allowed the subdivision into 5 groups, different for diagnostic and / or nosographic characteristics. In particular:

- $46 \%$ of the general population (23 subjects) were included in Group A "Affective immaturity and regulatory difficulties";

- $12 \%$ in Group B (6 samples) "Genetic syndromes";

- $18 \%$ in Group C (9 samples) "Language Delay and/or DSA";

- 12\% (6 samples) in Group D "Intellectual Disability and/or Psychomotor Developmental Delay";

- $12 \%$ (6 samples) in Group E "Social Adaptation Disorder".

\section{The administration of the TEC-I}

\section{"Test of understanding of emotions"}

The protocols of administration of the test provide for the use of 23 standardized monochromatic boards depicting expressive faces of male or female characters involved in different emotional states. In order to promote and support the analysis of the processes of reading, identification and emotional recognition, the qualitative choice of the cards to be used and of the sex of the character represented in them, was updated from administration to administration for each sample, in order to create correspondence of sex between the character of the cards presented by the examiner and the subject examined.

The tables were presented individually to each subject of each sample of the population according to a precise numerical order as per the manual. The administration, through the modalities reported by the manual, is aimed precisely at supporting the progressiveness and promoting the gradual emergence of the logical-solving reasoning, as well as stabilize the performance levels examined in itinere and finally give, during the initialling phase, a correct and truthful analysis of the general data taking.

The test includes a peculiar analysis of reading and of basic and complex emotional recognition: according to the procedures through the first items it is asked, with simple and closed verbal tasks, to recognize and 
identify instantly the basic emotional state of each character represented. In the second items, through the use of questions or stories exposed in a neutral tone, the comprehension, tuning and identification of the mental and emotional states of others, as well as the intentions, beliefs and unconscious desires of the different characters (ToM) are tested. At the end of the administration, the sealing procedures are appropriately performed through standardized conversions and calibrations. In fact, through the use of the manual the specific raw scores obtained for each emotional component probed (n9), are reported in the Response Sheet and subsequently analyzed according to the variables $p(z)$ or "standardized score" and p.c. or "percentile range" in the Coding Sheet. Through the $p(z)$ analysis it is possible to understand the standard deviation of each sample, i.e. how far the levels achieved have deviated from the average (equal to 1) and/or from the expected competences by age; while through the interactive and critical p.c. analysis the standardized score is contextualized and related in a precise percentile range. The p.c. analysis sometimes subverts the statistical deviation of the sample. In fact, while $p(z)$ distinguishes the performance outcomes collected according to three values: above average (green in the graphs shown), borderline (yellow) and below average (red), the percentile range - specific to age and type/number of component achieved - introduces a fourth value known as average (white). The delineation of the latter determines four types of ranges:

- a pathological one (below 5 p.c.);

- one borderline non-pathological but not even average (between $6^{\circ}$ and $24^{\circ}$ p.c.);

- one purely medium $\left(25^{\circ}\right.$ and $75^{\circ}$ p.c.);

- one above average (75th and 100 th p.c.).

\section{Data Analysis}

In the single analysis in p.c. and $p(z)$ for single group: Group A, consisting of 23 samples, according to the $\mathrm{p}(\mathrm{z})$ analysis $35 \%$ were above average, $26 \%$ below average and $39 \%$ in the borderline range. In the contextualized analysis in p.c. the subjects above the average are reduced to $14 \%, 41 \%$, about half of the subjects are in the average range, $32 \%$ belong to the borderline range and $13 \%$ are to be considered below the average.

$\mathrm{p}(\mathrm{z})$

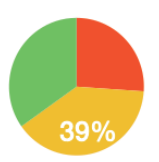

- Sotto la media

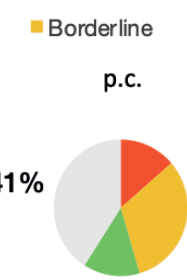

- Sotto la media

Borderline

- Sopra la media

nella media
The standardized scores obtained by group B and expressed according to the variable $p(z)$ are $17 \%$ above the average, $16 \%$ around the average, $67 \%$ below the average; however, taking into account the variable p.c. no sample is to be considered above the average, while only $17 \%$ are in the average standard. If according to the variable $p(z)$ only $16 \%$ could be considered belonging to the borderline range, according to p.c. it is half of the subjects and not $16 \%$ who can be placed in a borderline range and only half of the subjects expressed in $p(z)$ as pathological $(67 \%)$ could really be considered below the average (33\%).

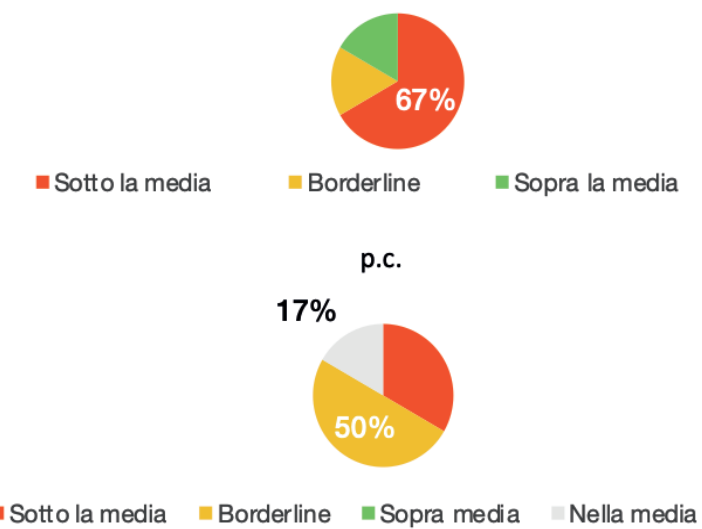

Graph. 2 - Group B

In Group C, the variable $p(z)$ allocated $44 \%$ of the subjects above the mean and $56 \%$ in the borderline range. According to the variable $p(c), 56 \%$ of the subjects were actually within the average range, and only $33 \%$ were borderline, while $11 \%$ were above average. Both variables, however, confirmed the absence of pathological or below average subjects.

$p(z)$

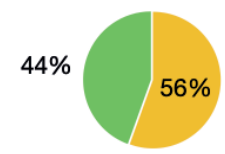

mSotto la media $\backsim$ Borderline $\backsim$ Sopra la media Nella la media p.c.

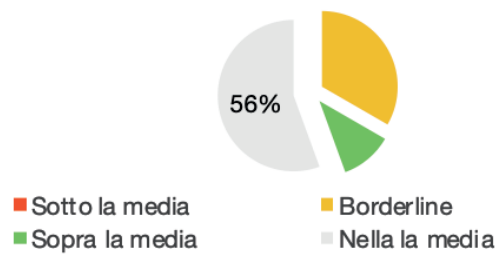

Graph. 3 - group $C$

The standardized scores obtained by group D and expressed according to the variable $p(z)$ are superimposable on those of group B: $17 \%$ of the samples are above average, $16 \%$ around average, $67 \%$ below average. The critical and interactive analysis in p.c. is similar and inverse: as for group B, no sample is above average and only $17 \%$ is in the average standard (between $25^{\circ}$ and $75^{\circ}$ ). However, $50 \%$ of the subjects are significantly below the average (in group B the same percentage was in a borderline range) while $33 \%$ are in the medium-low standards (in group B the same percentage was pathological). 
$\mathrm{p}(\mathrm{z})$

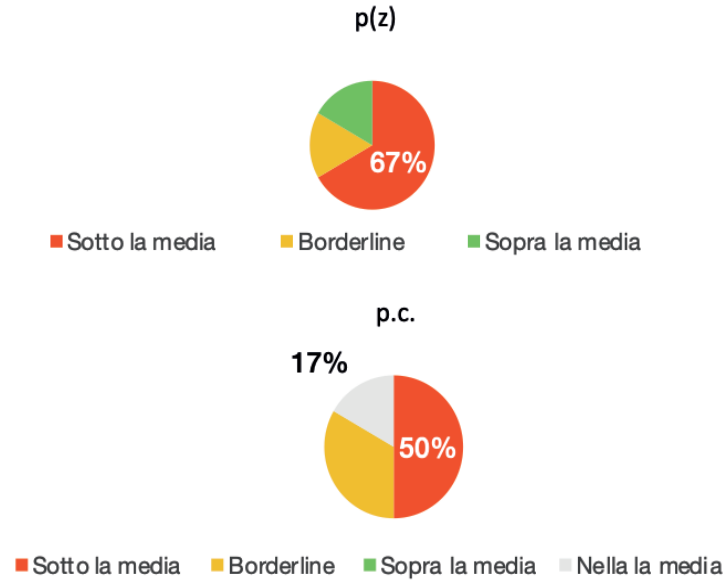

Graph. 4 - Group D charts

Finally, according to the variable $p(z), 83 \%$ of the samples in Group E are to be considered below average, while $17 \%$ belong to a borderline range. According to the variable $p(c)$, the borderline range increases up to $33 \%$ and the pathological range decreases up to $67 \%$. Both variables confirm the absence of subjects in the average range or above it.

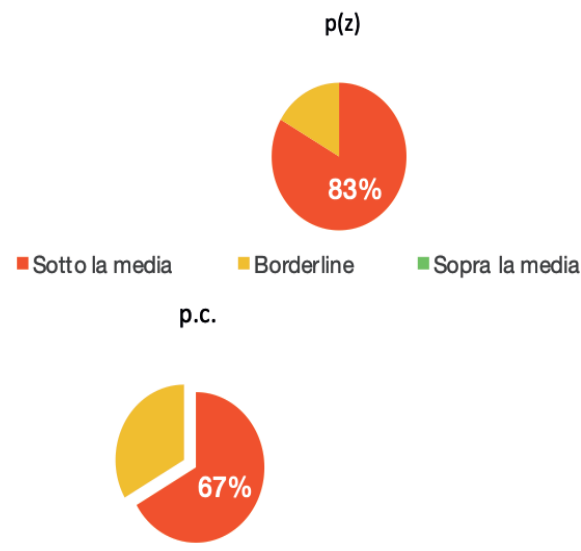

- Sotto la media $=$ Borderline $=$ Sopra la media $=$ Nella la media

Graph. 5 - Group E

\section{RESULTS AND DISCUSSION}

Regarding the General Population (n.50) according to the variable $p(z)$ the samples analyzed result to be $28 \%$ above average, $34 \%$ belonging to a borderline low average range and $38 \%$ below average. However, in the interactive and critical analysis according to the p.c. variable only the borderline sub- jects are really confirmed as such (34\%), the pathological samples are reduced to $24 \%$, those on the average increase to $32 \%$ and $10 \%$ are even above it. In the comparative analysis between Group A (n.23) and all the other groups B-C-D-E (n.27) significant data emerged. In fact, according to the variable $p(z)$ the subjects with a primary diagnosis of regulatory and/or psycho-affective immaturity, although they showed coherent and typical emotional profiles for the gap underlying the nosographic category of belonging, achieved globally a better performance than the subjects belonging to Groups B-C-D-E. The latter, clinically numbered in primary disorders of a different nature - genetic syndromes or anomalies, mild/ moderate intellectual disability, psychomotor developmental delay, expressive language disorders and/ or pragmatic components, social adaptation disorders and/or Autistic Spectrum - showed no less difficulties, albeit in secondary terms, than the subjects in Group A. On the emotional-affective level, in fact, they showed more borderline and significantly below average subjects and fewer profiles in the average or above average range. In fact, in statistical terms, according to the variable $\mathrm{p}(\mathrm{z})$ there were +7 subjects below average, +1 borderline and -2 samples above average. Also the variable p.c confirms the better statistical trend of the subjects of Group A: in groups $\mathrm{B}-\mathrm{C}-\mathrm{D}-\mathrm{E}$ there are +6 subjects below the average, +3 borderline, - 3 samples above the average and - 2 and in the average.

Although not all the data collected are useful for the mathematical calculation of the final standardized score, the entirety and completeness of the data confer statistical significance and reliability to the test. Moreover, experimental experience suggests a complete as well as progressive administration of the standardized material, even in the presence of clinical pictures visibly or clearly compromised.

As a matter of fact, despite the progression of the cards recalls the linear and typical development of the emotional components expected and achievable by age, the interruption of the test, because of the objective difficulty to learn complex components in the face of inadequate results on simple requests, is partially correct: some altered, inverse or elusive developmental trajectories associated with the clinical-pathological variability and the transversality of the emotional interferences in the dynamic processes of growth, sometimes outline singular, atypical and irregular statistical profiles. Some subjects, subverting the hierarchical learning of skills, have correctly
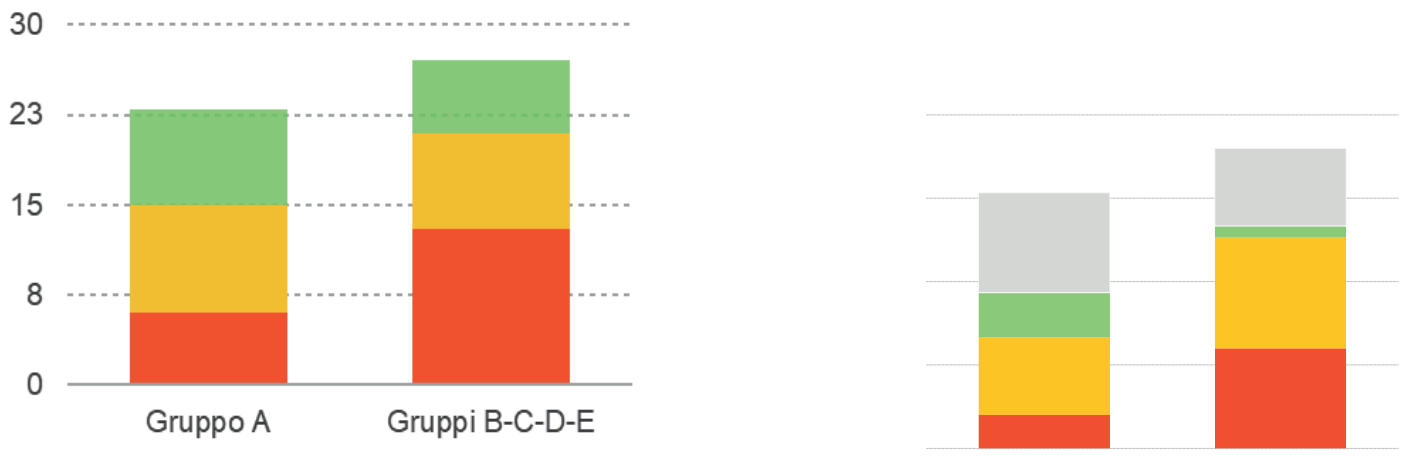

Graph. 6 - Comparative and interactive graphs according to variable p(z) and p.c. 
answered the last questions rather than the first ones, while others have surprisingly achieved results above or below the average, the limits and/or the nosographic expectations. The a priori knowledge of the different diagnostic algorithms and/or of the linearity of the stages of the emotional-affective development does not justify point by point the unpredictability or the divergence of the statistical results found and therefore does not present true data in the presence of a partial administration of the tables.

\section{REFERENCES}

1. Albanese and Paola Molina, The development of the understanding of emotions and its evaluation, Unicopli Edition (2013). Anolli, The emotions, Unicopli. (2003)

2. Anolli, Fundamentals of communication psychology, handbooks, il Mulino. (2012)

3. Arace, When children begin to. Childhood psychology and first steps in the development of the self, Mondadori University (2018).

4. Ainsworth, Infancy in Uganda: Infact care and the growth of love, Johns Hopkins University Press, Baltimore (1967).

5. Ainsworth, Attachments beyond infancy, in "American Psychologist",44,1989,pp.709-716.

6. Ainsworth, Some considerations regarding theory and assessment relevant to attachments beyond infancy, in M.T..

7. Bowlby, The influence of early environment in the development of neurosis and neurotic character, in "International Journal of Psycho-Analysis",21,1940,pp.154-178

8. Bowlby, Child care and the growth of maternal love, Penguin Books, London 1953.

9. Bowlby, Attachment and loss, vol.1: Attachment to the mother, Bollati Boringhieri, Turin, 1972.

10. Bowlby, Attachment and loss, vol. 2: Separation from the mother, Bollati Boringhieri, Turin, 1972.

11. Bowlby, Attachment and loss, vol. 3: The loss of the mother, Bollati Boringhieri, Turin, 1983 (ed.or.1980).

12. Bowlby, Construction and rupture of affective ties, R.Cortina, Milan, 1982 (ed.or.1979).

13. Bowlby, A secure base, R.Cortina, Milan, 1989 (or.ed.1988).

14. Brenner-R.Mueller, Shared meaning in boy toddler's peer relations, in Child Development, 53, 1982 , pp.380-391.

15. Camaioni, Paola Di Blasio, Psychology of development, Manuali, Il Mulino.

16. Ekman, Universal and cultural differences in facilitating expressions of emotions, in K.Cole, University Press, Lincoln, 1972.

17. Ekman, Darwin and facial expression: Acentury of research in review, Academic Press, New York,1973.

18. Gison, Bonifacio and Minghelli, Autism and psychomotricity, Erickson

19. Goleman Daniel, Emotional Intelligence, Bur Rizzoli.

20. Grazzani, Psychology of emotional development, Second edition, Il Mulino (2014)

21. Hewstone, M., Stroebe, W. Introduction to social psychology, Il Mulino, Bologna (2002)

22. Lindzey, Thompson, Spring, Psychology. Zanichelli (1991)

23. Relatives and Magda Arnold. Psychology of the emotions. Horizons of knowledge. (2017)

24. Plutchik. The Nature of Emotions. American Scientist. vol 89 (1980)

25. Plutchik. Psychology and biology of the emotions, Bollati Boringhieri

26. Stern, D. The interpersonal world of the child, Bollati Boringhieri (1987)

27. Ruggiero. Verduyn, P., Lavrijsen S. Which emotions last longest and why: The role of event importance and rumination. Motivation and Emotion, University of Leuven (2014).

28. Zorzi, M., V., Girotto, Fundamentals of general psychology, Il Mulino, Bologna. (2004) 